This item was submitted to Loughborough's Research Repository by the author.

Items in Figshare are protected by copyright, with all rights reserved, unless otherwise indicated.

\title{
Sensor-enabled PCBs to aid right first time manufacture through defect prediction
}

PLEASE CITE THE PUBLISHED VERSION

http://dx.doi.org/10.1109/EPTC.2014.7028396

PUBLISHER

(C) IEEE

VERSION

AM (Accepted Manuscript)

\section{PUBLISHER STATEMENT}

This work is made available according to the conditions of the Creative Commons Attribution-NonCommercialNoDerivatives 4.0 International (CC BY-NC-ND 4.0) licence. Full details of this licence are available at: https://creativecommons.org/licenses/by-nc-nd/4.0/

\section{LICENCE}

CC BY-NC-ND 4.0

\section{REPOSITORY RECORD}

Sharpe, Richard, Guy Banwell, Paul P. Conway, and Andrew A. West. 2019. "Sensor-enabled Pcbs to Aid Right First Time Manufacture Through Defect Prediction”. figshare. https://hdl.handle.net/2134/20217. 


\title{
Sensor-enabled PCBs to aid right first time manufacture through defect prediction
}

\author{
Richard Sharpe, Guy Banwell, Paul P. Conway, Andrew A. West \\ Loughborough University \\ Wolfson School of Mechanical and Manufacturing Engineering, Loughborough University, UK, LE11 3TU \\ Email: r.sharpe@lboro.ac.uk
}

\begin{abstract}
Prevention of defects can reduce waste and aid lean strategies such as right first time manufacture. The prediction of defects leads to prevention; however accurate prediction requires a high understanding of the domain and mechanics of each defect. For a prediction simulation to adapt to a manufacturing line's conditions requires timely information about the products being manufactured. In this paper, research into the addition of a sensory circuit to a PCB in order to monitor defects through its manufacture into a PCBA is outlined. Manual handling and the number of thermal cycles are attributors to many of a product's potential defects. The use of an accelerometer and temperature sensor in a circuit alongside a processor and RFID chip is presented. The use of RFID allows the board to communicate to the manufacturing line, increasing the current state of intelligence for this type of product. The use of an RFID chip also allows data storage for both manufacturing information as well as sensory information. This intelligence capability could be added to the PCB in one of two ways; embedding within the layers of the board or by integrating into a pallet or carrier which the PCB will be associated with throughout its manufacture.
\end{abstract}

\section{Introduction}

Electronics manufacture, specifically assembly, can be split into two distinct domains; High Mix Low Volume (HMLV) and Low Mix High Volume (LMHV). HMLV products are manufactured predominantly for the aerospace and defense domain. Customers of these products demand assurance of high build quality and that every finished product will function reliably. The products themselves are highly complex and comprise of valuable components. These reasons make the manufacture of these products relatively expensive as the costs invoked by any rework, prototype or pilot builds only have a small volume to be distributed across.

'Right-first-time' is a lean strategy which can be used in manufacturing; it is important to recognise defects and their causes for this to be achieved. To make a Printed Circuit Board Assembly (PCBA) right first time, defects would need to be prevented from occuring. If this can be achieved or even partly achieved it can directly reduce three recognised waste categories [1]; overproduction, extra processing steps (rework) and defects.

Enabling products, such as a PCBA, to communicate and begin to contribute to decisions which affect its destiny is an early stage in becoming an Intelligent Product [2]. A graph, published by Meyer [3], states the three key areas of product intelligence are; (i) the level of its intelligence capability, (ii) where this intelligence is located and (iii) its aggregation, i.e. can it communicate to other intelligent products which are assembled onto it.
At this time the highest level of intelligence given to PCBAs is 'Information Handling', the product is able to communicate information about itself [4], either via barcode or Radio Frequency Identification (RFID), (Fig.1). All the storage and processing of communications is done on the product, and is an intelligent entity which has no ability to directly communicate to what is assembled onto it.

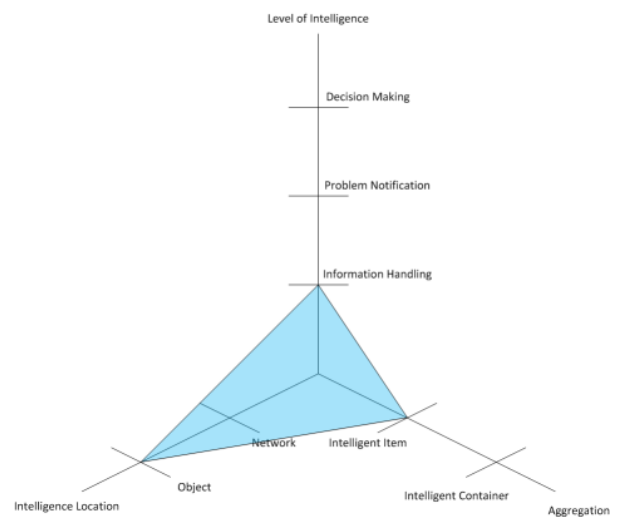

Figure 1 - Intelligent Product Graph, Current State of PCB

Previous research has reported the prediction of defects resulting from issues with the design of a Printed Circuit Board (PCB), particularly the layout of components, footprints and interconnections [5]. However a significant number of defects occur as a result of how the product is handled, stored and treated during the manufacturing processes. The next step for the research outlined in this paper is to combine realtime information collected from the manufacturing line and integrate it with defect prediction simulation software. This simulation would be run for each board at set manufacturing stages. The research presented is key in both getting the link to the product and the collection of the information. In the future it is envisaged that, like RFID which have successfully been embedded into boards (Fig. 2), sensors will also become part of the board, providing an ambient product and process monitoring system.

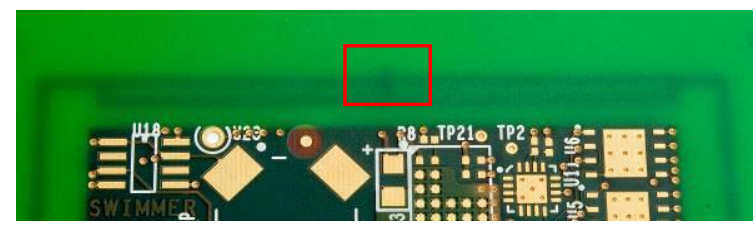

Figure 2 - Embedded RFID in PCB

The embedding of RFID has been shown in published research [6] and is also now available via commercial channels e.g. PCB-Pool, although it is still not prevalent in mass manufacture.

The research which is described in this project is centralised around the use of an RFID enabled manufacturing line. Manufacturing lines in other industries have been 
previously integrated and the authors, with colleagues, have constructed such a system for this domain, providing the capability to have two way communications with the product. The Ultra High Frequency (UHF) RFID traceability system constructed is integrated onto an operational Surface Mount Technology (SMT) line (Fig. 3). It currently records the process history of each unique product and is being extended to communicate with other objects such as the tooling and machines, supporting business processes through the real-time analysis of collected data.

The following work has progressed in multiple concurrent stages (i) design and prototype of the RFID with sensor circuit, (ii) manufacture on a single layer, (iii) validation of sensor information, (iv) embedding of circuit, (v) communication to board during manufacture.

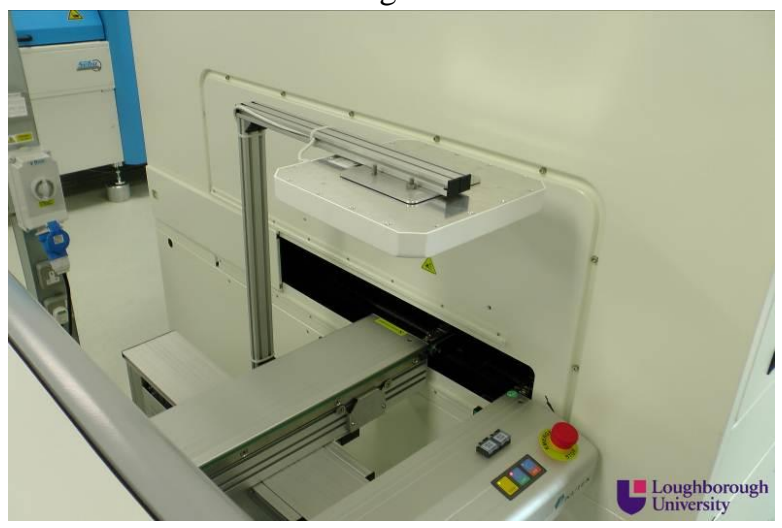

Figure 3 - RFID Implementation on SMT Line

\section{Defects Related to Product Experience}

There are many defects which can occur in electronics manufacturing. To go alongside this array of defects there is a large list of potential causes which can interact with each other.

Causes of defects can be linked to numerous different aspects of manufacturing, some of which can be attributed to the product itself. These experiences, as shown in Fig. 4, have been split into three categories; Handling, Environmental and Manufacturing Operations. 'Handling' describes how the product is moved around the facility. 'Environmental' relates predominantly to the temperature and humidity, for example, how many thermal cycles the product has been through. It can also be combined with the handling to determine proper storage of the product. The final category is 'Manufacturing Operations', this covers aspects such as which processes were carried out and which resources were employed.

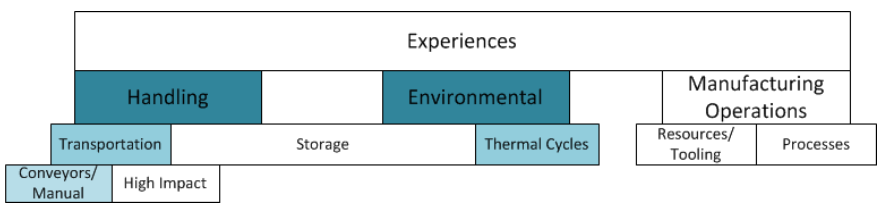

Figure 4 - PCBA Product Experiences

The experiences focused on in this research were recognition of the mode of transport and the number of thermal cycles of a product.

Research into faults found that manual handling of products greatly increased the risk of numerous defects.
Reasons for this were that, by manually handling a product, there was a higher risk of contamination, high vibrations and of being dropped. The defects caused by these events include, bridging, tombstoning, missing components and misalignment.

The number of thermal cycles is also a key contributor in component failure. The thermal cycle which a PCBA has to go through to reflow the solder paste reaches approx. 225$260{ }^{\circ} \mathrm{C}$. It is important to monitor the number of thermal cycles: too many thermal cycles reduces reliability of solder joints [7][8], can cause delamination [9] and also adversely affect the adhesive holding the pads down. It is highly likely that a PCBA would experience at least two thermal cycles in general manufacturing, as the majority of boards are double sided, but if the board also has Through Hole (TH) components then another thermal cycle will take place during wave soldering. It is important when deciding whether or not a product should be reworked that this count is borne in mind as reworking will involve another two thermal cycles; one to remove the component and one to replace.

\section{System Architecture}

The proposed system built around this technology is focused on the prediction and prevention of defects during manufacture. In electronics assembly, as in many other industries, the reduction of defects prevents waste, lowers rework operations, lowers manufacturing cycle time and maximizes efficiency of both line and operator utilisation.

To be able to simulate for prediction it is essential to retrieve up-to date data, these data must be accurate and complete. It is important that these data are retrieved without any lag; the longer the delay the more chance there is of predicting a defect for which the point of prevention has passed.

The overall system (Fig. 5) presented relies on a strong link to the product via UHF RFID, it is also able to communicate to resources and interchangeable machine parts via the same infrastructure and is networked directly to the manufacturing machines.

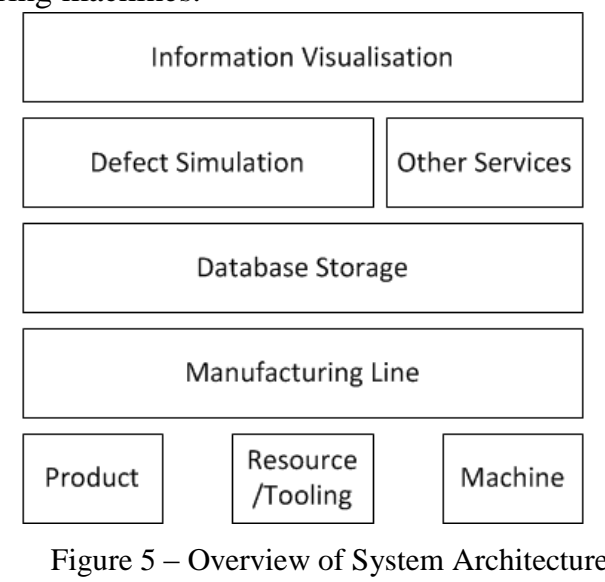

Through communication with the machines, the system can determine the various states of manufacturing operations and, via the RFID link, determine exactly which product is approaching, being processed, and exiting. Data collected can be compiled and stored in a database located either locally or remotely (depending on security requirements). It is currently proposed that at key events, such as process completion, alerts 
from the manufacturing line would be sent to trigger a defect simulation to be executed. The way in which the data is stored allows multiple simulations to be run, extended and adapted without affecting the recording of information.

An important part of such a system is how the end user would visualise the information/knowledge derived. Two methods exist, one is to show the information on GUIs the other is to show it on the product itself, for example the flashing of LEDs.

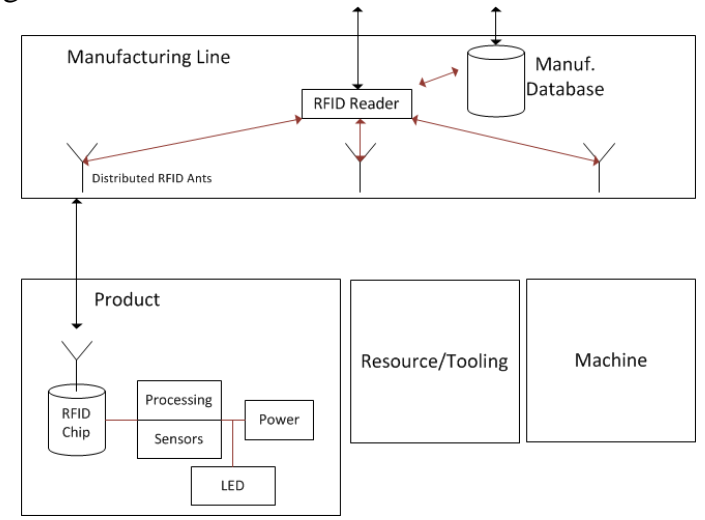

Figure 6 - Product to Manufacturing Line Overview

The areas of the system which are presented and discussed in this paper are the product and manufacturing line entities (Fig. 6).

The product will communicate to the line via its passive UHF RFID chip. To detect the experiences desired the product will require sensors and the ability for limited processing which, in turn, demands a power source. The manufacturing line will contain multiple, distributed UHF RFID readers each with multiple antennae providing coverage in key areas of the facility.

\section{Intelligent, Sensor Enabled PCB Design}

The circuit layout to provide the intelligence to the PCB comprises of an accelerometer, temperature sensor, humidity sensor, microprocessor, RFID chip and matching antenna. At this point it was externally powered.

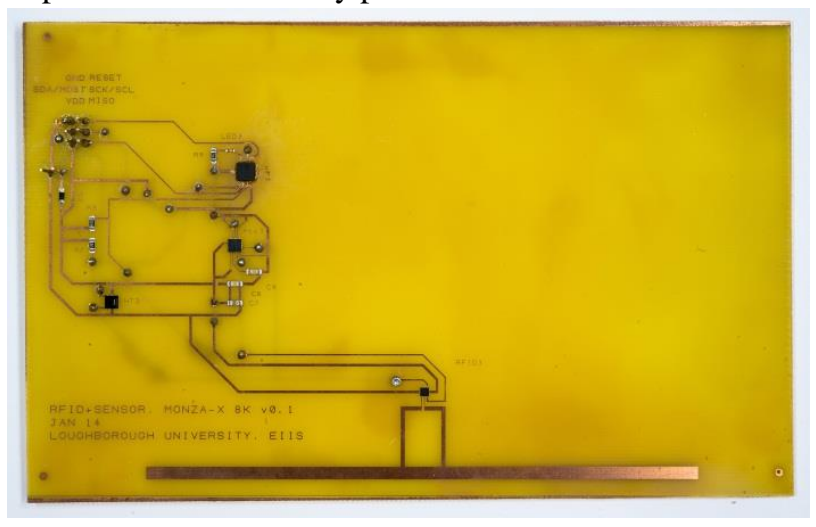

Figure 7 - Sensory Circuit, Prototype

The initial prototype was made at Loughborough University (Fig. 7) with the aim to test the communication between components, this was then optimized for surface area coverage and built by industrial collaborators (Fig. 8).

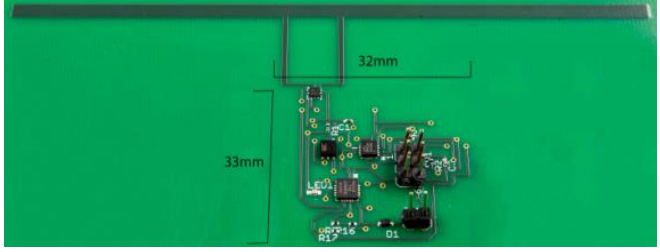

Figure 8 - Sensory Circuit v2, Prototype

\section{Sensor Validation and Characterisation}

\section{Manual transportation vs Conveyor}

The initial prediction for trying to characterise the method of transportation had been that monitoring of the $\mathrm{Z}$ axis of the board would be sufficient, using a threshold value to limit processing requirements (Fig. 9). The results, however, showed that the biggest difference was in the $\mathrm{X}$ axis, the direction of the conveyor (Fig. 10).

The test was carried out with a sampling rate of $50 \mathrm{~Hz}$. The conveyor used was adjustable width, belt driven and supported the board along two edges, it was controlled by light gates at the far ends of travel (Fig. 9).

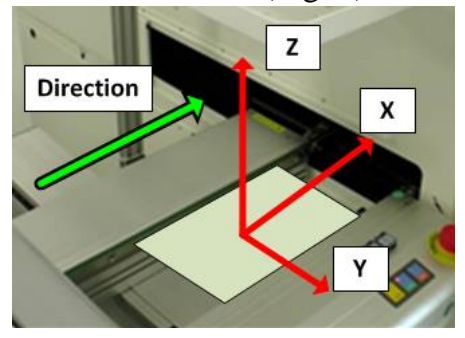

Figure 9 - Accelerometer alignment

The results from this show that, to correctly compare between being manually transported from the process and transportation via conveyor, monitoring of the $\mathrm{X}$ axis for approximately $0.5 \mathrm{~s}$ is required (Table 1 ). The sampling rate required to pick up the peak would have to be higher than $12.5 \mathrm{~Hz}(1 / 0.08 \mathrm{~s}$, average time above threshold), to get an accurate representation 3 data points should be used, approximately $40 \mathrm{~Hz}$ ensuring recognition. The threshold value from these results was initially indicated at $0.15 \mathrm{~ms}^{-2}$, this should ensure a sample is taken within the $0.5 \mathrm{~s}$ window which will indicate the product's transportation method.

It is not possible to confidently distinguish these modes via the other 2 axes (Fig. $11 \& 12$ ).

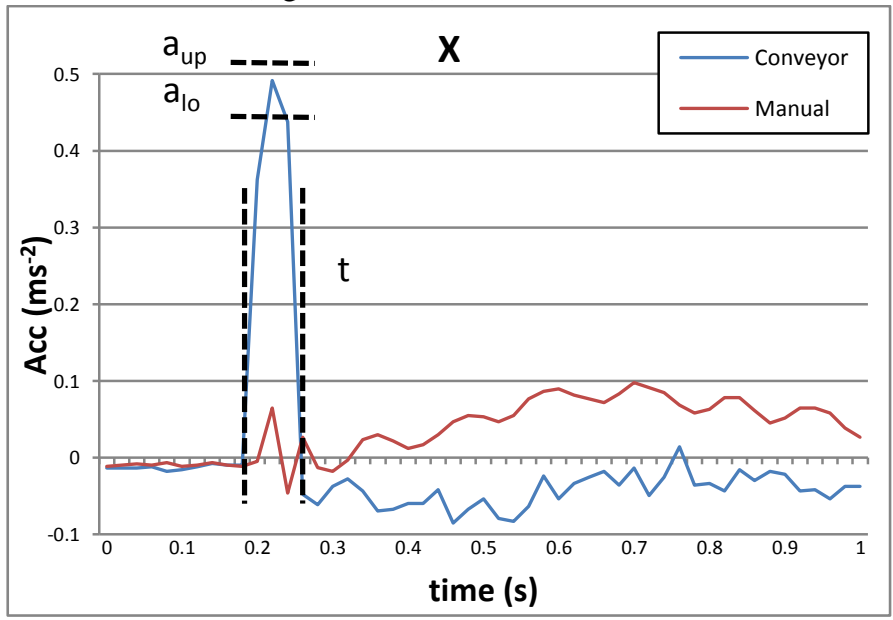

Fig 10 - X Axis Accelerometer Trace 


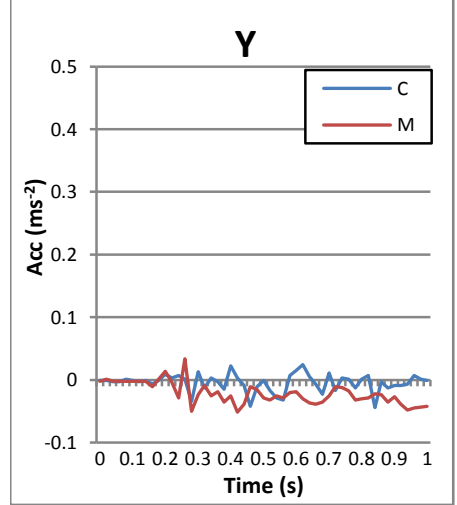

Fig 11 - Y Axis Accelerometer Trace

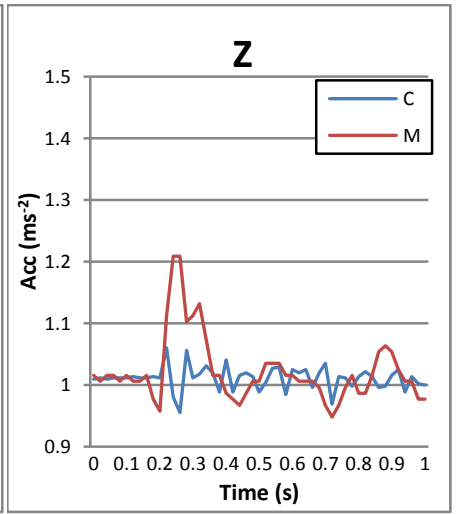

Fig 12 - X Axis Accelerometer Trace
Table 1 - Threshold Values, X direction, accelerations

\begin{tabular}{|c|c|c|c|}
\hline Feature & Symbol & Value & Unit \\
\hline Max acc upper & $\mathrm{a}_{\text {up }}$ & 0.51 & $\mathrm{~ms}^{-2}$ \\
\hline Max acc lower & $\mathrm{a}_{\mathrm{lo}}$ & 0.46 & $\mathrm{~ms}^{-2}$ \\
\hline Threshold acc & $\mathrm{a}_{\mathrm{th}}$ & 0.15 & $\mathrm{~ms}^{-2}$ \\
\hline Avg time higher above threshold & $\mathrm{t}$ & $0.08 \mathrm{~s}$ & $\mathrm{~s}$ \\
\hline
\end{tabular}

\section{Thermal Cycling}

It was predicted that the circuit would not function in the high temperatures during reflow. The temperature sensor was stated to operate up to $125^{\circ} \mathrm{C}$ whilst the processor had an operation stated at $85^{\circ} \mathrm{C}$ and a limit of $125^{\circ} \mathrm{C}$.

The board was able to record temperatures in excess of $100^{\circ} \mathrm{C}$ before degradation in performance (approximately $110^{\circ} \mathrm{C}$ ). Upon cooling down the device was able to operate again.

These initial trials took the board to $150^{\circ} \mathrm{C}$, lower than that of reflow.

Huang et al concluded from their research that one of the key influences on damage to component packing via delamination was the total time above $100^{\circ} \mathrm{C}$ and temperature during this [9]. It would be extremely unlikely for a board on an SMT manufacturing line to experience a temperature above that of $100^{\circ} \mathrm{C}$ and not continue to one much closer to reflow.

\section{Proposed Operation}

The ability to characterise experiences of a product, in this case a PCBA, is vital to predict complex defects. For this to be achieved it is important to research the procedure for such a process. A board sampling the proposed sensors at a high rate would demand a high energy requirement. Different strategies for monitoring will therefore have to be evaluated upon product completion to ensure power efficiency.

To determine how a product has been transported between processes only requires a board to be monitored during this transition. The amount of time to monitor has been investigated in the sensor validation and characterisation section.

The authors' proposed operation for manual handling detection would therefore be as follows; a product finishes a process, the machine alerts the system which in turn sends a signal to the board via the RFID infrastructure. The board, upon receiving the signal, would then begin monitoring its accelerometer to determine its mode of transportation. The duration for which the board monitors its movement would have to be further researched for the features to be recognised. Initially, to evaluate whether it has been picked up, only a couple of seconds would be required. However, it could be argued that it is also the putting back down of the product and the vibrations seen throughout its manufacture that would better help to evaluate the risk of a defect.

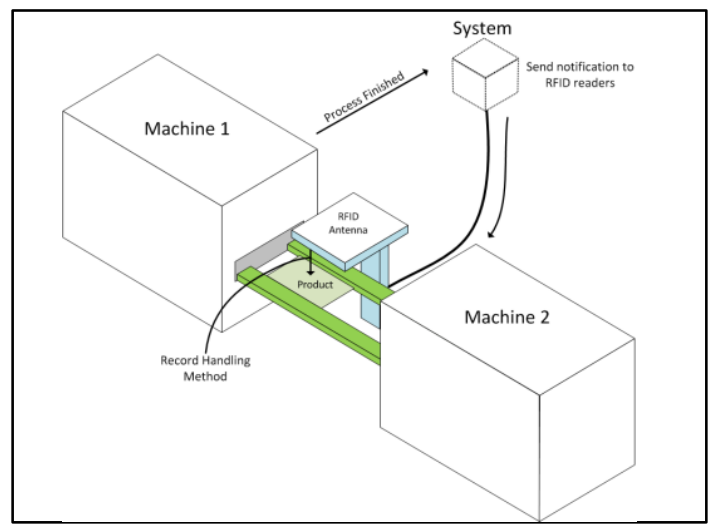

Figure 13 - Proposed Operation

The signal sent to the PCB's RFID chip would most likely be in the form of a 'write' function performed by the RFID reader, changing a value inside the memory which the processor is monitoring.

\section{Full Embedding Of Intelligence}

The initial processes in electronics assembly require the board to have a flat surface. If the intelligence hardware is to be located on the product then it should arrive from the PCB manufacturer with the capacity of intelligence assembled. This would enable successful defect prediction from receipt of goods.

An initial part of this research involved the embedding of LEDs within the inner layers of PCBs (Fig. 14). Following this success it is the aim to embed the circuit which has been validated in the presented results. The area of research is centred around finding the limitations of embedding SMT components i.e. component height limits, component placement methods and component positioning.

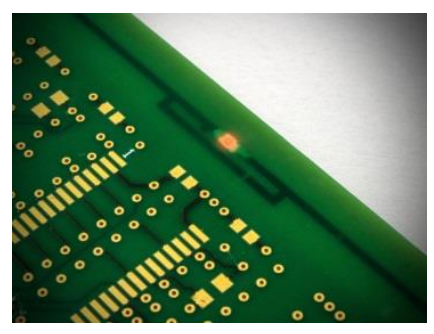

Figure 14 - Embedded LED

When a PCB is made, it is initially made as a group of boards, on one larger board called a panel. This is then split up after board manufacture, either into individual boards or smaller numbers depending on size for the assembler to use. 
Fig. 15 shows the sensor board panelised for manufacture. An array of LEDs is also to be embedded on this build to further progress the initial research in component survival.

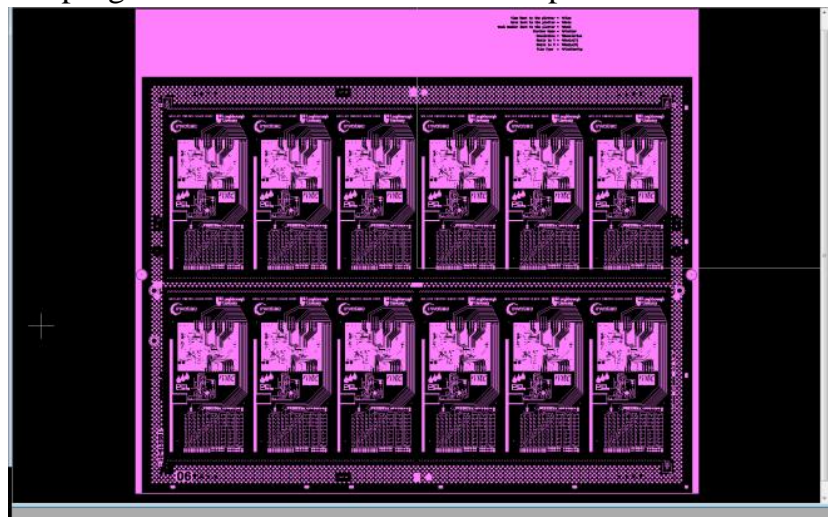

Figure 15 - Panelised Design

Fig. 15 is just one layer in a multiple layer board and will have to be bonded to subsequent layers. This is done under high pressure and temperature and it is possible that different areas of the panel may experience varying conditions. The embedding of the sensor circuit and LED array will greatly contribute to any design rules needed for the future of embedded components.

\section{Conclusions \& Current Limitations}

This paper has demonstrated that an accelerometer monitoring a PCB can determine how it has been transported, and subsequently help to predict defects. Specifically the axis of acceleration in the direction of conveyor movement has to be monitored. The research has also shown that a temperature sensor could be used within the same circuit to monitor the number of thermal cycles of the board.

The research has shown that experiences which are proven contributors to the occurrence of defects can be captured, stored and relayed by the product itself.

More experiences will now be investigated to increase the impact this could have on an electronics assembler's waste. These include correct storage, impacts (drops) and indirectly the monitoring of machines.

Current limitations in adding the capability of intelligence to a PCBA are power and survival of the circuit in extreme conditions such as those experienced during reflow.

The embedding of the circuit is an area of research which will require a substantial amount of work before it is accepted by industry. However it is not the only way to give a PCBA intelligence. Commonly in electronics assembly a product is manufactured on a standard sized pallet (otherwise known as a carrier) as this eliminates the time spent adjusting conveyor widths. A solution which could be deployed is to embed the intelligence into the pallet. This would make packaging easier and allow thermal protection for both the power source and processor. It would also mean the investment would stay inside the manufacturing firm and would not necessarily have to add any cost to the final product. It does however limit the potential to aid the rest of the supply chain, which, for electrical components, goes all the way to Waste Electronic and Electrical Equipment (WEEE) recycling and back to market.
Referencing the first chart shown (Fig. 1), which displayed the current product intelligence capacity for a PCB, two more charts can now be plotted (Fig. 16). Both of these show a board which can sense its experiences, The yellow area (Fig. 16a) shows how a product which communicates to the system and can effectively carry out simulations about itself over a network achieves a high level of intelligence [10]. It is now able to take part in the decision making which influences its destiny. If, for example, it has been through a high number of thermal cycles it could either alert or not allow a machine to process it for fear of irreversible damage. The green triangle (Fig. 16b) illustrates that if the product is left as an entity and does not have the ability to perform processing off-board, it can no longer predict but it can have simple process rules embedded to allow problem notification. For example the LED could flash when the number of thermal cycles is high, and go solid when the maximum recommended number has been reached. These concepts would support defect prediction, lowering waste and aid right first time manufacture, ultimately making a firm more competitive.

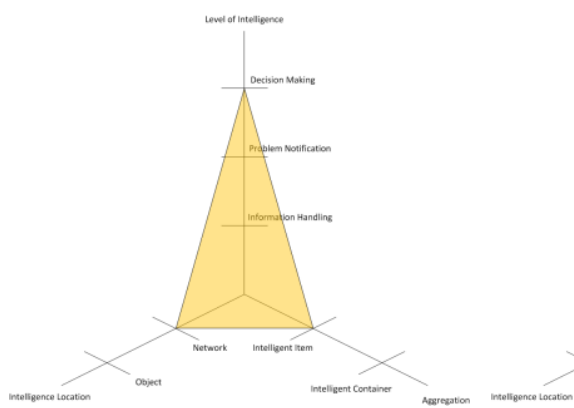

(a)

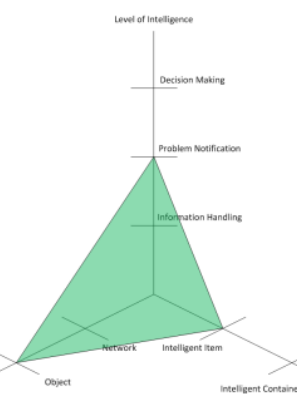

(b)
Figure 16 - Intelligent PCB Potential

The future goals for this research are not only to enable product intelligence in the electronics manufacturing domain but to research how this will improve a manufacturer's efficiency and positively affect their business processes.

\section{Acknowledgments}

The authors would like to thank the Technology Strategy Board (TSB) for the funding of project "INTELLICO" and EPSRC for the project "AIIM", grant reference EP/K014137/1.

\section{References}

1. Jones, D.T. et al "Lean logistics." International Journal of Physical Distribution \& Logistics Management, Vol 27, Issue 3/4 (1990), pp. 153-173.

2. McFarlane, D. et al, "Auto ID systems and intelligent manufacturing control" Engineering Applications of Artificial Intelligence, 16 (2003), pp. 365-376.

3. Meyer, G. G. et al, "Intelligent Products: A survey" Computers in Industry, 60 (2009), pp. 137-148.

4. Bindel, A. et al, "Information structure required for lifecycle monitoring of electronic products" Institution of Mechanical Engineers, Part B: Journal of Engineering Manufacture, 226 (2012), pp. 1612-1627. 
5. Bindel, A. et al "New lifecycle monitoring systems for electronic manufacturing with embedded wireless components", Circuit World, Vol 36 Iss 2, pp. 33-39.

6. Quintero, L. A. H. et al "Integrated modelling for simulation in the electronics manufacturing domain" Proc $11^{\text {th }}$ Electronics Packaging Technology Conf, 2009, pp. 260-266.

7. Nurmi, S.T. et al "The influence of multiple reflow cycles on solder joint voids for lead-free PBGAs", Soldering \& Surface Mount Technology, 15:1 (2003), pp. 31-38.

8. Guo, F. et al "Effects of reflow on wettability microstructure and mechanical properties in lead-free solders" Journal of Electronic Materials, Vol 29, Issue 10 (2000), pp. 1241-1248.

9. Haung, Y.E. et al "Effect Of Solder Reflow Temperature Profile On Plastic Package Delamination" $23^{\text {rd }}$ Electronics Manufacturing Technology Symposium (1998), pp. 105-111.

10. Yang, X. et al, "Intelligent products: From lifecycle data acquisition to enabling product-related services" Computers in Industry, 60 (2009), pp. 184-194. 